\title{
A Prospective Double-Blinded Randomized Controlled Study Comparing the Efficacy of a Novel Biodegradable Synthetic Polyurethane Foam (Nasopore) vs Standard Polyvinyl Acetate Sponge (Merocel) as Packing Material after Functional Endoscopic Sinus Surgery: The First Indian Experience
}

${ }^{1} \mathrm{~S}$ Raghunandhan, ${ }^{2}$ Mohan Kameswaran, ${ }^{3} \mathrm{~J} o h n \mathrm{~K}$ Thomas

\begin{abstract}
Background: In Indian clinical practice, conventional nasal packing for hemostasis after routine rhinological surgery is usually performed with Vaseline (paraffin) gauze, and rarely with glove-finger packs or tamponade balloons. These materials are tedious to pack and cause discomfort to the patient on removal. Newer nasal packs which have recently emerged in the Indian scenario are found to be more user-friendly, equally effective for hemostasis and less traumatic to the operated nasal mucosa. Most rhinologists today, prefer to use polyvinyl acetate sponge packs (Merocel/Ivalon) for tamponade after nasal surgery. These packs are very effective but nonabsorbable and need to be removed which does not augur well with many patients postoperatively. The recent entry of a biodegradable synthetic polyurethane foam (Nasopore) as an alternative nasal packing material, has evoked new interest, which initiated this study.
\end{abstract}

Study method: This prospective randomized double-blinded controlled study was aimed to compare the clinical efficacy and patient comfort level, while using Merocel and Nasopore as packing material after functional endoscopic sinus surgery (FESS). This study included thirty adults who were diagnosed with moderate to severe bilaterally comparable chronic rhinosinusitis, who underwent FESS under general anesthesia and received size-matched nasal packs randomly - Merocel on one side and Nasopore on the other. The assessment of clinical efficacy of both packs with regards to ease of packing, hemostasis, pressure effects, infections and adhesions was done with a Diagnostic Nasal Endoscopy at first postoperative day, first week and fourth week after surgery. All Merocel packs were removed on the first postoperative day. Patient comfort levels for both packs were recorded with a standard symptom questionnaire marked on a visual analogue scale of ten and the results were statistically compared between the two groups.

Results: Comparable outcomes were found while using Merocel or Nasopore with regards to ease of nasal packing

\footnotetext{
${ }^{1,2}$ Consultant and ENT Surgeon, ${ }^{3}$ Senior Registrar

${ }^{1-3}$ Department of Rhinology, Madras ENT Research Foundation, Chennai, Tamil Nadu, India
}

Corresponding Author: S Raghunandhan, Consultant and ENT Surgeon, Department of Rhinology, Madras ENT Research Foundation, Chennai, Tamil Nadu, India, Phone: 04424311411, e-mail: raghunandhansampath@gmail.com and control of postoperative bleeding. There was a statistical difference in the hemostatic property between the two materials in the immediate postoperative period. Five out of 30 patients developed reactionary bleeds with Nasopore, which required repacking with same material within the first 24 hours, but no further bleeds were noted. Two out of these five patients on the first postoperative day had migration of Nasopore toward the choana and had to be repacked with additional Nasopore. Sequential postoperative nasal endoscopy revealed that Nasopore is more mucosal friendly with lesser incidence of adhesions, synechiae, infection and edema, with better biocompatibility and safety. The major success with Nasopore was found to be, the fact that no pack removal was necessary, which immensely improved patient satisfaction and willingness to use the material when compared to Merocel. This was proved by the patient's symptom questionnaire which showed significant benefits of Nasopore over Merocel with regards to compliance and comfort levels.

Conclusion: Nasopore is a novel biodegradable synthetic material which is clinically as efficacious and patient-friendly as Merocel and is suitable for postoperative nasal packing after functional endoscopic sinus surgery. The clinical benefits of Nasopore and its outcomes among patients as recorded in our study, stands proof to support Nasopore as a successful packing material in rhinological surgery.

Keywords: Chronic rhinosinusitis, Functional endoscopic sinus surgery, Nasal packing, Merocel, Nasopore.

How to cite this article: Raghunandhan S, Kameswaran M, Thomas JK. A Prospective Double-Blinded Randomized Controlled Study Comparing the Efficacy of a Novel Biodegradable Synthetic Polyurethane Foam (Nasopore) vs Standard Polyvinyl Acetate Sponge (Merocel) as Packing Material after Functional Endoscopic Sinus Surgery: The First Indian Experience. Clin Rhinol An Int J 2014;7(3):105-111.

Source of support: Nil

Conflict of interest: None

\section{INTRODUCTION}

As per standard clinical practice, chronic rhinosinusitis which has been refractory to comprehensive treatment with topical and oral medications over a period of 3 weeks or more necessitates surgical intervention for adequate disease clearance and establishment of ventilation and 
drainage for the paranasal sinuses. Functional endoscopic sinus surgery (FESS) has today become the norm in such cases providing successful results world-over. A spectrum of nasal packing materials is now available for achieving hemostasis and for prevention of synechiae/adhesions in the postoperative period. Patients undergoing FESS in the Indian scenario are conventionally packed with Vaseline (paraffin) gauze for achieving postoperative hemostasis. To a lesser extent, a custom-made glovefinger pack or tamponade balloons are also used. Even though the traditional Vaseline gauze packing provides successful tamponade of epistaxis in the postoperative period, it is tedious to pack postoperatively if the patient has recovered from anesthesia and is conscious. Most patients feel pain, pressure and discomfort while packing and on removing the pack on the first postoperative day.

Newer polyvinyl acetate sponge packs (Merocel/ Ivalon) have come into the Indian market, which are more patient friendly and are equally effective for hemostasis and less traumatic to the operated nasal mucosa. The most popular nasal pack used currently in our practice is Merocel (Medtronic, Xomed Surgical Product, Jacksonville, Florida, USA), a nonabsorbable polyvinyl acetate sponge pack which needs to be removed using a draw-string postoperatively. The recent entry of a novel biodegradable, synthetic polyurethane foam (Nasopore, Polyganics BV, Groningen, The Netherlands) as an alternative nasal packing material, has evoked immense interest among Rhinologists across the globe, who have begun using this material for a spectrum of nasal surgeries and early results are now emerging from their experiences.

There is no Indian literature available as yet on Nasopore, with regards to its clinical efficacy and patient satisfaction, or comparing it with other standard packing materials. This triggered the need for this study and we share our first Indian experience which would initiate further clinical research on this interesting biodegradable packing material in the future.

\section{Study Method}

This prospective randomized double blinded controlled study was aimed at comparing the clinical efficacy and patient comfort level while simultaneously using Merocel and Nasopore as packing materials. This clinical study was conducted at the Rhinology Clinic of Madras ENT Research Foundation, which is a premier ENT institute and Tertiary Care Referral Centre in Chennai, South India. This study was cleared by the institutional ethical review board in June 2012. It was devised as a doubleblinded randomized controlled trial in which two coinvestigators participated in various ways to complete the study. The patients included into the study were coun- seled in detail regarding the use of two different nasal packs and their properties and an informed consent was taken. Patients were also explained regarding the symptom questionnaire prior to their participation in the study.

This study included adult patients, aged between 21 and 63 years $(\mathrm{M}: \mathrm{F}=2: 1)$, diagnosed with chronic rhinosinusitis refractory to comprehensive treatment with topical and oral medications over a period of 3 weeks. The severity of chronic rhinosinusitis was assessed as per the standard Lund Mackay CT scan scoring system and the Lanza Kennedy Endoscopic grading system. ${ }^{1}$ Patients included as per the above scoring systems had comparable bilateral moderate to severe sinusitis with symmetrical ostiomeatal complex (OMC) obstructions and infundibular disease, which required surgical intervention with functional endoscopic sinus surgery under general anesthesia. The exclusion criteria for the study, was presence of severe rhinosinusitis involving all the paranasal sinuses associated with sinonasal polyposis, allergic fungal rhinosinusitis, gross septal deviation or spurs hitching on the OMC region, huge concha bullosa or allergic turbinate hypertrophy in contact with septum. Postoperative recurrent rhinosinusitis, patients with bleeding diathesis, patients on aspirin or anti-platelet drugs and hypertensive patients were excluded from this study.

All patients were blinded regarding on which side what nasal pack was placed after surgery. Patients needed to share their experience on the symptom questionnaire on the first postoperative day after undergoing their first diagnostic nasal endoscopy, and again after nasal endoscopy at the first week and fourth week after surgery. The operating surgeon was not involved in the study and he selected each side of the nose randomly for packing with Merocel or Nasopore with no bias. Both the size-matched Nasopore (configuration: Standard $4 \mathrm{~cm}$ ) and Merocel (custom-made to $4 \mathrm{~cm}$ along with a draw-string used for removal), were injected with Gentamicin $80 \mathrm{mg}$ for providing local expansion to the materials and for providing topical antiseptic effect. The same surgeon performed all FESS surgeries using the standard technique of anterior and posterior ethmoidectomy, frontal sinusotomy and middle meatal antrostomy. He then placed both the Nasopore and Merocel in the middle meatus lateral to the middle turbinate, using the same method with endoscopic assistance. The surgeon reflected on the comfort of placement of the nasal pack and its immediate hemostatic effect to the investigators of this study after surgery.

All patients were provided with oral antibiotics and intranasal saline douches from the first postoperative day. Pack removal on the Merocel side was done by the operating surgeon on the first postoperative day and 
subsequently the patient was referred to the investigators for follow-up. The investigators were blinded from the intraoperative findings, and they were responsible only for assessment of the clinical efficacy and patient compliance of the materials postoperatively. The first investigator conducted sequential diagnostic nasal endoscopy at the first day, first week and fourth postoperative week to assess the endoscopic status of the nasal packing with regards to nasal bleeding, mucosal injury and healing status, middle meatal synechiae, infection, granulation, adhesion, and any migration of pack over the period of follow-up. A grading scale from 0 to 3 was created for assessment of the severity of each of the above signs, which were documented meticulously at each schedule with nasal endoscopy (Fig. 1).

The second investigator analyzed the patient symptom questionnaire with no knowledge about the intra or postoperative endoscopic findings in these patients. This questionnaire was based on the standard visual analog scale (VAS), wherein score ' 0 ' meant no symptom and score ' 10 ' meant unbearable symptom. This VAS questionnaire evaluated the various aspects of patient comfort with respect to pain, pressure, nasal block, dysphagia, sleep disturbance, postnasal drip, allergic symptoms, general satisfaction and willingness to use the material (Table 1). The second investigator was also involved with the statistical analysis of data by paired ' $t$ '-test method using SPSS 17.0 statistical software and he compared and interpreted the results between the two groups.

\section{OBSERVATIONS AND RESULTS}

\section{Evaluation of Clinical Efficacy}

In our study, comparable outcomes were found while using Merocel or Nasopore with regards to ease of nasal packing and control of immediate postoperative bleeding. The operating surgeon felt that both Nasopore and Merocel had equal ease while packing and possessed similar tensile strength after injection with Gentamicin, and hence both provided an equal amount of support in the middle meatus, lateral to the middle turbinate. There was no statistical difference in the hemostatic property between the two materials in the immediate postoperative period (Table 2).

With regards to their hemostatic property, patients with Merocel packs 26/30 patients (86.6\%) provided excellent hemostasis until they were removed on the first postoperative day, while, $3 / 30$ patients $(1 \%)$ had minimal nasal bleeding confined to the nose and $1 / 30$ patients $(0.3 \%)$ had moderate nasal bleeding which did not require repacking. On the Nasopore side, we had successful hemostasis among $21 / 30$ patients (70\%), 4/30 patients (13.3\%) had mild to moderate bleeding which did not need repacking while $5 / 30$ patients $(16.7 \%)$ needed repacking with Nasopore, due to reactionary hemorrhage. Among these 5 patients, 2/30 (6.6\%) had developed bleeding due to migration of the Nasopore material from the middle meatus, posteriorly toward the choana and repositioning these displaced packs with additional Nasopore packing helped secure the hemostasis, while the other 3 patients $(1 \%)$, had bleeding in spite of the Nasopore being in proper position and they required repacking with an additional Nasopore for arresting the bleeding, probably due to inadequate tamponade effect from the Nasopore standard configuration which was used for this study. In such cases we feel that the Nasopore forte configuration would be more appropriate due to its stronger hemostatic property. We did not experience any further bleeds after this intervention within the first postoperative day and we did not encounter any secondary hemorrhage due to infection later on with either Merocel or Nasopore. The degradation of Nasopore which was sequentially monitored with endoscopy, did not cause any delayed or rebound bleeds over the next 2 weeks, and the degraded Nasopore material did not further get displaced into the nasopharynx to cause any signs of aspiration.
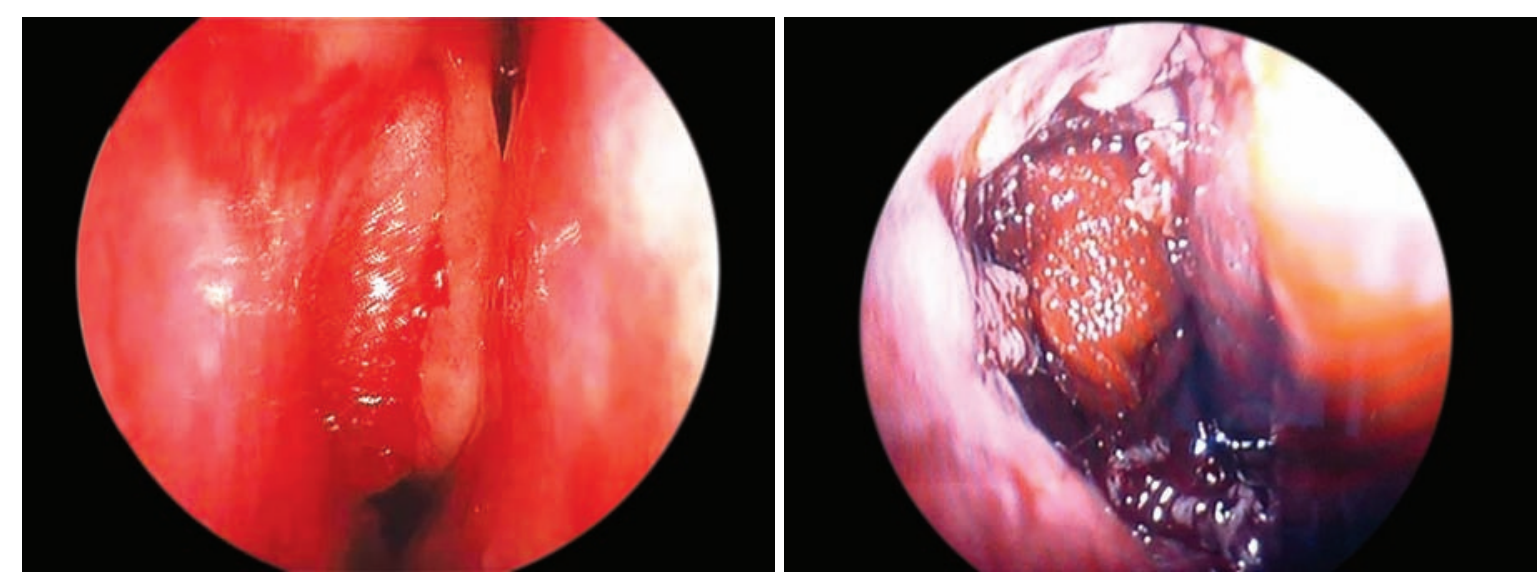

Fig. 1: Endoscopic picture of right middle meatus showing Nasopore in situ as on 1st and 7th POD 
Table 1: Visual analog scoring sheet for patient symptoms

\begin{tabular}{lllllllllllll}
\hline Symptoms & Merocel side & Nasopore side & \\
\hline Pain during pack removal & 012345678910 & 0 & 1 & 2 & 3 & 4 & 5 & 6 & 7 & 8 & 9 & 10 \\
Pain/pressure effect due to packing & 012345678910 & 0 & 1 & 2 & 3 & 4 & 5 & 6 & 7 & 8 & 9 & 10 \\
Nasal discharge & 012345678910 & 0 & 1 & 2 & 3 & 4 & 5 & 6 & 7 & 8 & 9 & 10 \\
Nasal obstruction & 012345678910 & 0 & 1 & 2 & 3 & 4 & 5 & 6 & 7 & 8 & 9 & 10 \\
Difficulty in swallowing & 012345678910 & 0 & 1 & 2 & 3 & 4 & 5 & 6 & 7 & 8 & 9 & 10 \\
Sleep disturbance & 012345678910 & 0 & 1 & 2 & 3 & 4 & 5 & 6 & 7 & 8 & 9 & 10 \\
Postnasal drip & 012345678910 & 0 & 1 & 2 & 3 & 4 & 5 & 6 & 7 & 8 & 9 & 10 \\
Allergic symptoms & 012345678910 & 0 & 1 & 2 & 3 & 4 & 5 & 6 & 7 & 8 & 9 & 10 \\
General satisfaction & 012345678910 & 0 & 1 & 2 & 3 & 4 & 5 & 6 & 7 & 8 & 9 & 10 \\
Willingness to use & 012345678910 & 0 & 1 & 2 & 3 & 4 & 5 & 6 & 7 & 8 & 9 & 10 \\
\hline
\end{tabular}

$(0=$ No symptom $\longrightarrow 10=$ Severe symptom $)$

Table 2: Grading of perioperative nasal bleeding

\begin{tabular}{llll}
\hline Bleeding & Grade & $\begin{array}{l}\text { Merocel } \\
(n=30)\end{array}$ & $\begin{array}{l}\text { Nasopore } \\
(n=30)\end{array}$ \\
\hline $\begin{array}{l}\text { No bleeding } \\
\text { Minimal-confined to nose }\end{array}$ & 1 & 26 & 21 \\
$\begin{array}{l}\text { Moderate-bleeds out of } \\
\text { nose }\end{array}$ & 2 & 3 & 3 \\
$\begin{array}{l}\text { Severe-requires } \\
\text { repacking* }\end{array}$ & 3 & 0 & 1 \\
\hline
\end{tabular}

*p-value $<0.05$ with $95 \%$ confidence interval

Sequential postoperative nasal endoscopy revealed that Nasopore is more mucosal friendly with lesser incidence of adhesions, synechiae and edema. This was statistically proven when the endoscopic findings were graded and prospectively compared between both the nasal packs (Table 3). The overall mean average endoscopic grading for adhesions/synechiae was in support of the Nasopore group. The incidence of infection was comparable between both groups. Aggressive infections at the site of nasal packing, due to either of these materials was found to be negligible, possibly due to the use of topical gentamicin in these packs. We found no untoward allergic reactions to either of the materials or any foreign body reaction with granulation formation at the site of packing. Hence, we could conclude that both these nasal packs were safe, biocompatible and effective for achieving adequate hemostasis and tamponade after nasal surgery.

\section{Evaluation of Patient Outcomes}

The major success with Nasopore was found to be, the fact that no pack removal was necessary, which immensely improved patient compliance, satisfaction and willingness to reuse the material when compared to Merocel. This was proved by the sequential assessment of patient's symptom questionnaire on a VAS overtime, which showed significant benefits of Nasopore over Merocel with regards to compliance and comfort levels. A comparative analysis of the VAS scores by paired ' $t$ '-test method showed statistically significant benefits in support of Nasopore over Merocel (p-values $<0.05$ with $95 \%$ confidence interval) on first postoperative day (Table 4). None of the patients showed willingness to re-use Merocel again, while $21 / 30$ patients $(70 \%)$ were willing to try Nasopore in the future. $9 / 30$ patients $(30 \%)$ were averse to using any nasal pack ever again, since they were also averse to the undergoing any further surgical procedure overall.

Nasopore was not removed unlike Merocel and hence pain on removal could not be compared in our study. While comparing other symptoms on the VAS questionnaire, we did not find statistically significant differences between the two groups. The mean average score for pressure symptoms on the day of surgery was found to be comparable. Patients had significant rhinorrhea and

Table 3: Postoperative endoscopic grading (7th postoperative day)

\begin{tabular}{|c|c|c|c|}
\hline Adhesion & Grade & Merocel $(n=30)$ & Nasopore $(n=30)$ \\
\hline No adhesion & 0 & 21 & 27 \\
\hline Mild-easy to release* & 1 & 9 & 3 \\
\hline Moderate-hard to detach & 2 & 0 & 0 \\
\hline Severe-needs synechiae release & 3 & 0 & 0 \\
\hline Infection & Grade & Merocel $(n=30)$ & Nasopore $(n=30)$ \\
\hline No infection & 0 & 25 & 28 \\
\hline Mild infection & 1 & 5 & 2 \\
\hline Moderate infection & 2 & 0 & 0 \\
\hline Severe infection & 3 & 0 & 0 \\
\hline
\end{tabular}

${ }^{*} p$-value $<0.05$ with $95 \%$ confidence interval 
Table 4: Comparative analysis of patient symptom score on 1st postoperative day

\begin{tabular}{lll}
\hline Mean average VAS & Merocel & Nasopore \\
\hline Pain during pack removal & $7.4 \pm 0.7$ & 0 \\
Pain/pressure effect due to & $5.4 \pm 0.9$ & $4.6 \pm 1.2$ \\
nasal pack & & \\
Nasal discharge & $5.5 \pm 1.0$ & $3.6 \pm 0.8$ \\
Nasal obstruction & $7.6 \pm 0.8$ & $7.2 \pm 0.6$ \\
Difficulty in swallowing & $1.6 \pm 0.7$ & $1.2 \pm 0.6$ \\
Sleep disturbance & $2.9 \pm 0.4$ & $2.6 \pm 0.6$ \\
Postnasal drip & $3.5 \pm 0.5$ & $3.9 \pm 0.6$ \\
Allergic symptom & $2.1 \pm 0.6$ & $1.6 \pm 0.6$ \\
General satisfaction* & $2.8 \pm 1.0$ & $8.2 \pm 1.2$ \\
Willingness to use* & $2.4 \pm 1.0$ & $7.8 \pm 1.1$ \\
\hline
\end{tabular}

${ }^{*} p$-value $<0.05$ with $95 \%$ confidence interval

postnasal drip on the Merocel side after pack removal on the first postoperative day, while persistent nasal block on the Nasopore side at the same time, but this difference was mostly attributable to the act of pack removal on the Merocel side, rather than as a result of the biomaterial used. We did not record complaints of allergy, vomiting, indigestion, dysphagia, choking or foreign body sensation among our patients due to the Nasopore kept in situ. We did record complaints of snoring among 3/30 of our patients $(10 \%)$ in the first postoperative week, but there was no predilection as to which side of their nose was blocked more, thus inducing noisy breathing. Hence, Nasopore could not be implicated as the cause for their snoring and sleep disturbance.

\section{DISCUSSION}

Functional endoscopic sinus surgery is a continuously evolving field that has had many exciting developments in the past three decades. Advances in mucosal-sparing techniques have achieved better wound healing with rapid postoperative re-epithelialization and reciliation. Many surgeons believe that the postoperative treatment regimen is as important as the surgery itself, since the ultimate goal of FESS is to re-establish normal mucociliary clearance in the sinuses. All sinus surgeons have the common objective of achieving excellent hemostasis and postoperative healing that avoids adhesion formation and lateralization of the middle turbinate. Nasal packing has been the traditional method of controling ongoing bleeding after sinus surgery. The use of removable nasal packs like Vaseline gauze or Merocel is still widely followed worldwide. There are several advantages of these removable nasal packs like they are cheap, easy to manipulate and align within the nose, and provide better supporting ability to a trimmed middle turbinate. ${ }^{2}$ But, there is a small incidence of complications documented in literature with these removable nasal packs like infections, septal perforation, pack migration and aspiration, toxic shock syndrome, allergies, foreign body granuloma and obstructive sleep apnea. ${ }^{3}$ But, patients feel that the most unpleasant experience of nasal surgery is when they undergo pack removal.

The advent of newer biomaterial based nasal packs like the absorbable porcine gelatin (Surgiflo, Ethicon Inc, Somerville, New Jersey), impregnated with thrombin, topical antifibrinolytic epsilon-aminocaproic acid (Amicar, Lederle Parenterals Inc, Carolina, Puerto Rico) or tranexamic acid (Cyklokapron, Pfizer, Puurs, Belgium) preparations, are diverse in their constituents and multicentric trials are presently underway to evaluate their clinical efficacy and outcomes. Such, absorbable nasal packs contain bovine or human blood clotting products to exploit the intrinsic and extrinsic coagulation cascades for producing hemostasis, which considerably reduces postoperative nasal bleeds while avoiding the bad experience of pack removal among patients.

Nasopore (Polyganics BV, Groningen, The Netherlands) is one such packing material which has shown promising results. It is a novel biodegradable, synthetic polyurethane foam which has been CE marked and FDA approved. It has widespread use among rhinologists of the European union since the last 2 years, and recently it has ventured into the Indian market. The main advantage of Nasopore over other commercial nasal packs is that it is bioresorbable, biologically inert and after the healing process it drains out from the nose in the form of water, carbon dioxide and polyamines within 2 weeks. Its unique structure enables rapid and high volume fluid absorption which swells, molds and conforms to the anatomical shape of the middle meatus. Topical application of steroids or antibiotics on the Nasopore helps in improved mucosal healing, which has also been proved and routinely practiced while using Merocel nasal packs. The Nasopore standard is available in 4 and $8 \mathrm{~cm}$ sizes and has a porosity of 97 to $98 \%$ with fast degradation and high absorption within 2 weeks time. Nasopore is also available as Forte and Forte Plus, which have higher density, better supporting ability and compressibility with lower porosity (93-97\%) and slower absorption rates (3-4 weeks), which may be necessary while packing patients after surgery for extensive sinonasal polyposis, CSF leak repairs or excision of nasal tumors.

Prabhu V et $\mathrm{al}^{4}$ (2007) and Franklin JH et $\mathrm{al}^{5}$ (2007), had extensively studied the impact of foam based absorbable nasal packs in patients undergoing FESS, and both their results highlighted the clinical safety and efficacy of absorbable biomaterials used as middle meatal stents in rhinological surgery. Shoman $\mathrm{N}_{\text {et }} \mathrm{al}^{6}$ (2009) were one of the first researchers to document the clinical safety and patient benefits while using Nasopore as a packing 
material. In their prospective, double-blinded, randomized trial using Nasopore as a middle meatal spacer in functional endoscopic sinus surgery, they highlighted its success in terms of hemostasis, wound healing and patient compliance. Our experience with Nasopore, agrees with Shoman et al, with regards to its clinical safety and its efficacy as a hemostatic agent. In our series, all Merocel packs provided excellent hemostasis until they were removed on the first postoperative day, with no further bleeds. On the Nasopore side, we had successful hemostasis among $25 / 30$ patients $(83.3 \%)$, while $5 / 30$ $(16.7 \%)$ patients needed repacking with Nasopore, due to reactionary hemorrhage. Among these 5 patients, 2/30 $(6.6 \%)$ had developed bleeding due to migration of the Nasopore material from the middle meatus posteriorly toward the choana and repositioning the displaced packs helped secure hemostasis, while the other 3 patients, required repacking with an additional Nasopore for arresting the bleeding. We did not experience any further bleeds after this intervention on the first postoperative day and we did not encounter any secondary hemorrhage due to infection later on. The possible reason for displacement of Nasopore could have been the fact that excessive soakage of this material due to blood and mucous could have enhanced the biodegradability of the surface layer of the pack, which loses its supportive strength and thus migrated out place. This phenomenon was not noticed in Merocel packs, possibly due to the fact that there is no biodegradation on the surface and also these packs are secured by tagging with a draw-string plastered over the nose which keeps it undisplaced.

Yoo Suk Kim et al ${ }^{7}$ (2011), recently performed a randomized single-blinded controlled study on the efficacy of Nasopore vs Merocel, when used in patients after septoplasty. They found that, there was no significant difference between Nasopore and Merocel, in terms of their hemostatic properties, while packing or re-packing the nasal cavities after surgery. They reported that Nasopore was more user-friendly in terms of ease of nasal packing, and the pain and bleeding while packing were lesser with Nasopore. Hence, these authors opined that patient's general willingness and acceptance to re-use Nasopore was significantly higher with Nasopore than with Merocel, which required to be removed subsequently. In our study, comparable results were obtained. Our results showed that the amount of mucosal injury while packing and further healing, when using either of these materials was indeed similar, even though there was a relatively higher incidence of crusting and granulation formation among the Merocel side, possibly since an additional frictional force acts on the mucosa while these packs are removed. Hence, we believe that both Nasopore and Merocel were equally user-friendly and efficacious as packing materials, but the main scoring point for Nasopore over Merocel is that it need not be removed postoperatively. This information when conveyed to patients during counseling prior to surgery enhances their willingness to use this material as they prefer to avoid the unpleasantness and anxiety, which is present during postoperative pack removal. Their satisfaction while using this material has also been documented sequentially with symptom questionnaires at answered on the 1st postoperative day, 1st week and 4 weeks later. Most patients do not feel the presence of any Nasopore material in their nose by the 4th week of follow-up, since it gets absorbed within 2 weeks duration, without any symptom sequelae.

In a recent study, Peter J Catalano et $\mathrm{al}^{8}$ (2011) clinically evaluated Nasopore for its safety and tolerability as a middle meatal stent, while comparing it to MeroGel in a series of 104 patients. They found comparable results between the two materials with higher incidence of infections on the MeroGel side. On the Nasopore side, they found no pain or allergic reactions. At follow-up till 16 weeks postoperatively, they did not encounter excessive bleeding, middle meatal synechiae or granulations while using Nasopore. They opined that the Nasopore material was either absent or negligible at 2 weeks postoperatively, by which time it is fully absorbed. They furthered their study onto the next phase which evaluated Nasopore as a drug-eluting middle meatal spacer, when impregnated with depot steroid preparation. They found Nasopore showed benefits as a successful drug-delivering system for at least 2 weeks, when placed in the middle meatus following surgery. Their ongoing research in a larger sample is targeted further to use Nasopore as a drug delivery technology which would obviate the need for systemic medications following nasal surgery.

A large number of evidence based clinical studies are underway and results are now emerging to prove that biomaterials impregnated with drugs are the best way forward in the treatment of sinus mucosal disease. Recent studies evaluating the efficacy of biodegradable drug-eluting middle meatal stents have been published by Weitzel EK, Peter J Wormald et $\mathrm{al}^{9}$ (2008) and Rowan Valentine et al (2009), ${ }_{1}^{10}$ but an overall consensus on a standard protocol regarding which nasal packing material is optimal and which topical drug regimen is most effective for intranasal use, is yet to be derived. Nasopore may possibly be one such drug-eluting biodegradable intranasal material in the near future. 


\section{CONCLUSION}

Nasopore is a novel biodegradable synthetic material which is clinically as efficacious and user-friendly as Merocel and is suit for postoperative nasal packing after functional endoscopic sinus surgery. The beneficial effects of Nasopore and the patient compliance with this material as recorded in our study stands proof to support Nasopore as a successful packing material in rhinological surgery. The authors strongly support the use of Nasopore over Merocel for its single-most advantage of biodegradability, which negates the need for pack removal thus alleviating significant anxiety and pain among patients in the postoperative period. The authors recommend the use of Nasopore in all basic nasal surgeries like septolasty, turbinoplasty, FESS, conchoplasty, synechiae release, etc. wherein this material has proven to be safe and effective. In due course this material will have wider usage and acceptance in the Indian scenario, if it is more cost-effective. Further studies are necessary to evaluate its use in extensive nasal surgeries for sinonasal polyposis, allergic fungal rhinosinusitis, nasal tumors and CSF rhinorrhea. Nasopore is an appropriate packing material for the future which heralds the concept of minimally invasive rhinological surgeries with maximal mucosal preservation.

\section{DISCLOSURE}

This clinical study was performed independently by the investigators with no liaison, influence or financial support from any company manufacturing nasal packing materials. The investigators have no conflict of interest with any companies or other similar research work. The results of this clinical study are solely the findings as recorded and interpreted by the investigators and they hold the responsibility for the inferences derived therein.

\section{REFERENCES}

1. Lund VJ, Kennedy DW. Staging for rhinosinusitis. Otolaryngol Head Neck Surg 1997 Sep;117(3 Pt 2):S35-S40.

2. Garth R, Brightwell A. A comparison of packing materials used in nasal surgery. J Laryngol Otol 1994 Jul;108(7):564-566.

3. Maccabee MS, Trune DR, Hwang PH. Effects of topically applied biomaterials on paranasal sinus mucosal healing. Am J Rhinol 2003 Jul-Aug;17(4):203-207.

4. Prabhu V, Kaushik V, Rhodes S, Tay H. Foam nasal packs: a prospective, randomized, patient-controlled study. Rhinology 2007 Sep;45(3):242-247.

5. Franklin JH, Wright ED. Randomized, controlled, study of absorbable nasal packing on outcomes of surgical treatment of rhinosinusitis. Am J Rhinol 2007 Mar-Apr;21(2):214-217.

6. Shoman N, Gheriani H, Flamer D, Javer A. Prospective, double-blind, randomized trial evaluating patient satisfaction, bleeding, and wound healing using bio-degradable synthetic polyurethane foam (Nasopore) as a middle meatal spacer in functional endoscopic sinus surgery. J Otolaryngol Head Neck Surg 2009;38:112-118.

7. Kim YS, Kim YH, Kim NH, Kim SH, Kim KR, Kim KS. A prospective, randomized, single-blinded controlled trial on biodegradable synthetic polyurethane foam as a packing material after septoplasty. Am J Rhinol Allergy 2011 MarApr;25(2):e77-e79.

8. Catalano PJ, Payne S, Thong M. Clinical evaluation of a fully synthetic middle meatal stent for safety and tolerability. Otolaryngol-Head Neck Surg 2011;144(3):452-456.

9. Weitzel EK, Wormald PJ. A scientific review of middle meatal packing/stents. Am J Rhinol 2008 May-Jun;22(3):302-307.

10. Valentine R, Wormald PJ, Sindwani R. Advances in absorbable biomaterials and nasal packing. Otolaryngol Clin North Am 2009 Oct;42(5):813-828. 